\title{
Design, development and performance optimization of a new artificial intelligent controlled multiple-beam optical scanning module
}

\begin{abstract}
This research presents a new approach to optimise the performance of a multiple-beam optical scanning system in terms of its marking combinations and speed, using Genetic Algorithm (GA). The problem has been decomposed into two sub problems; task segregation, where the marking tasks need to be segregated and assigned for each scanner head and path planning where the best combinatorial paths for each scanner are determined in order to minimise the total motion of marking time. The knowledge acquired by the process is interpreted and mapped into vectors, which are kept in the database and used by the system to guide its reasoning process. The main motivation for this study is to introduce and evaluate an advance new customized GA. Comparison results of different combinatorial operators and tests with different probability factors are shown. Also, proposed are the new modifications to existing crossover operator called DPPC (Dynamic Pre-Populated Crossover) together with modification of a simple crossover selection method, called BCS (Bi-Cycle Selection Method). The performance of the new operator called GA_INSP (GA Inspection Module) for a better evolutionary approach to the time-based problem has been discussed in the study. The representation approach has been implemented via a computer program in order to achieve optimized marking performance. This algorithm has been tested and implemented successfully via a dual-beam optical scanning module. (c) 2006 Asian Network for Scientific Information.
\end{abstract}

Keyword: Genetic algorithm; Multiple-head optical scanner 